\title{
Follow up study of cardiac involvement in Emery-Dreifuss muscular dystrophy
}

\author{
M YOSHIOKA, ${ }^{*} \mathrm{~K}$ SAIDA, $\dagger$ Y ITAGAKI, $\ddagger$ AND T KAMIYA§ \\ ${ }^{*}$ Department of Paediatrics, Kobe General Hospital, Kobe; Departments of + Neurology and $\ddagger$ Paediatrics, \\ Utano National Hospital, Kyoto; and §Department of Paediatrics, National Cardiovascular Centre, Osaka, \\ Japan
}

SUMmARY A patient with Emery-Dreifuss muscular dystrophy was followed up from 9 to 26 years of age. Serial electrocardiographic studies showed changes ranging from early cardiac conduction abnormality as first degree atrioventricular block to sick sinus syndrome. In addition the development of cardiomyopathy was documented by electrocardiographic, echocardiographic and radionuclide studies of the heart.

Emery-Dreifuss muscular dystrophy is a rare $\mathrm{X}$ linked condition clinically distinct from Duchenne and Becker muscular dystrophies. ${ }^{1}$ Onset is in childhood and cardiac involvement with conduction disturbances is a frequent and serious complication with a risk of sudden death. ${ }^{2}$

We encountered a 9 year old boy with characteristics of this disorder who later developed cardiomyopathy. Serial electrocardiographic, echocardiographic, and radionuclide studies were useful for estimating the degree of conduction disturbance and cardiomyopathy and predicting the prognosis. Although there are many reports of conduction disturbance in Emery-Dreifuss muscular dystrophy, ${ }^{2}$ serial studies of cardiac involvement are rare. We describe a follow up study of our patient from 9 to 26 years of age.

\section{Case report}

A 9 year old boy was admitted to Utano National Hospital for motor disability. There was neither consanguinity nor a history of neuromuscular disorders in his family. He was born at 35 weeks' gestation by vaginal delivery after a normal pregnancy, and his birth weight was $1900 \mathrm{~g}$. His motor milestones were delayed and he was unable to walk unaided until $2 \frac{1}{2}$ years of age. His intellectual development was normal. A waddling gait was noticed at the age of 4 years and he underwent Achilles tenotomy for relief of severe talipes equinovarus at 8 years of age. Muscle biopsy specimens obtained at that time from the quadriceps and gastrocnemius muscles showed a pronounced variation in the fibre size associated with many central nuclei, moderate perimysial fibrosis, and fatty infiltration.

Physical examination on admission showed abnormal neck posture and limited neck flexion, difficulty in climbing stairs, elbow contracture, and muscle wasting with a predilection for the shoulder girdle and peroneal muscles. Deep tendon reflexes could not be elicited. The serum creatine kinase activity was slightly raised. His heart rate was 73 beats/minute (bpm) and an electrocardiogram showed a first degree atrioventricular block with a PR interval of 0.20 seconds (figs $1 \mathrm{a}$ and $2 \mathrm{a}$ ).

At age 17, bradycardia was recognised in the early morning. An electrocardiogram showed atrial standstill with junctional rhythm at a rate of $40 \mathrm{bpm}$ and QS pattern in V2 (figs 1b and 2b). Cardiac catheterisation at that time showed normal ventricular performance. Cardiac output was $3.48 \mathrm{l} / \mathrm{minute} / \mathrm{m}^{2}$. An electrophysiological study showed elongated sinus recovery time up to $3388 \mathrm{msec}$; this is evidence of sick sinus syndrome. ${ }^{3}$ An echocardiogram also showed normal heart chamber size and performance with a left ventricular ejection fraction of $83 \%$ by the method of Pombo et al. ${ }^{4}$ A demand type cardiac pacemaker was implanted at the chest wall. An endomyocardial biopsy specimen from the right ventricle disclosed a variation in fibre size and focal fibrosis, suggesting the presence of cardiomyopathy (fig 3). An electrocardiogram at age 19 showed QS pattern in V1 to V3 and negative T in II, III, aVF and V3-V6 (figs 1c and 2c). His subsequent course was uneventful and he was discharged from the hospital at the age of 22 years and was able to work part time. At age 24 he developed signs of heart 
714 Yoshioka, Saida, Itagaki, and Kamiya

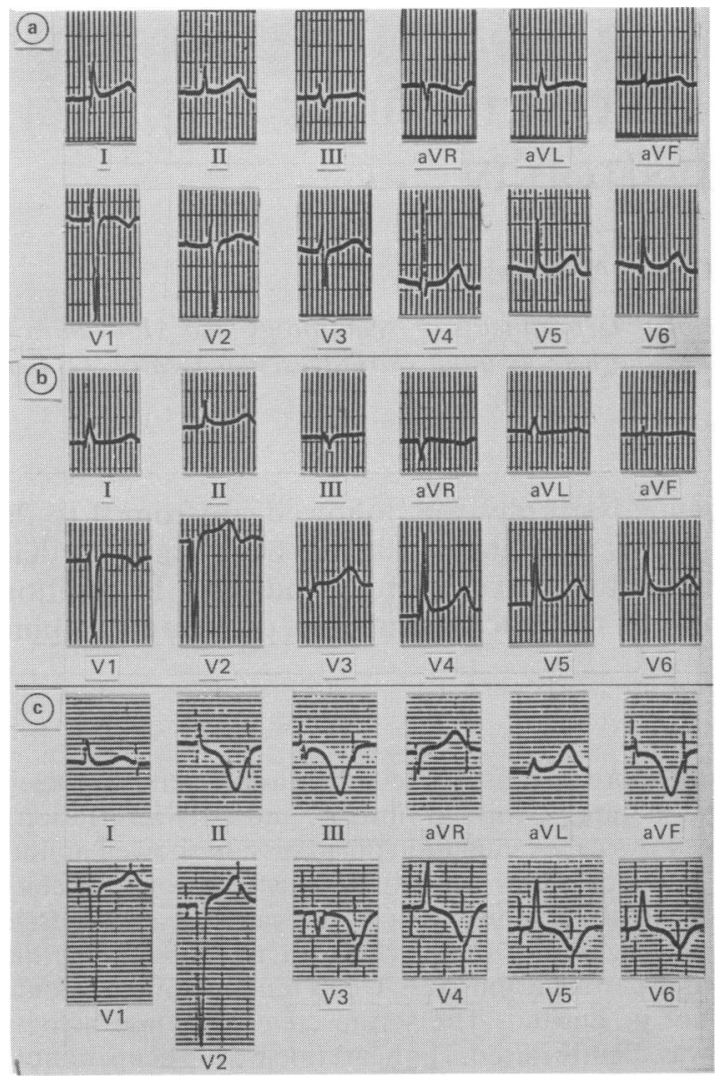

Fig 1 Serial electrocardiograms: (a) at 10 years 4 months of age, showing the first degree atrioventricular block with $P R$ interval of 0.20 seconds. (b) At 17 years 2 months of age, showing atrial arrest with junctional rhythm. QS pattern in V2 and $Q$ wave in V3 were also observed. (c) At 19 years 10 months of age after implantation of a permanent pacemaker. His own beats were shown. No atrial electrical activity was present and QS pattern spread from V1 to V3. Negative T in II, III, aVF and V3-V6 can also be seen.

failure and digitalis and diuretics were prescribed. An echocardiogram at age 25 showed a left ventricular ejection fraction of $66 \%$ by the method of Pombo et al. Gated blood pool scanning showed a dilated left ventricle with a left ventricular ejection fraction of $35 \%$ and generalised left ventricular hypokinesis with apical akinesis. Thallium imaging disclosed a pronounced perfusion defect of the cardiac muscle from the apex to the posteroinferior wall. Re-examination at the age of 26 showed a larger perfusion defect (fig 4). On the other hand motor disability of the patient developed slowly, and he was ambulant until 24 years of age. He was

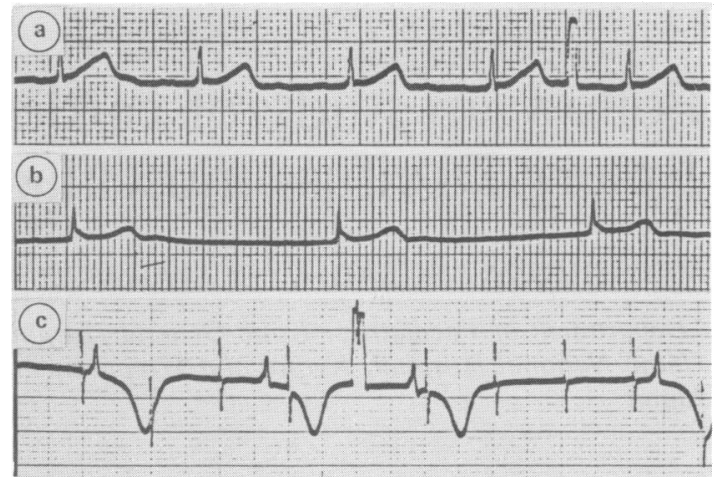

Fig 2 Longer rhythm strips of $(a),(b)$, and (c) in fig 1; (a) showing sinus rhythm, but (b) and (c) showing nodal rhythm.

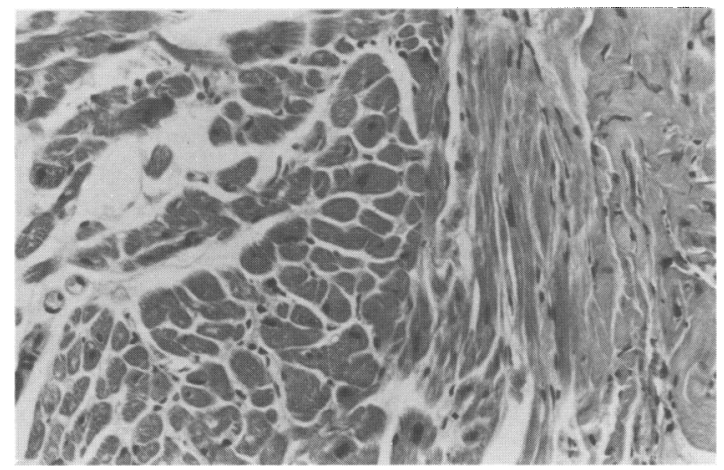

Fig 3 An endomyocardial biopsy specimen of the right ventricle showing a variation in fibre size. Focal fibrosis can be seen on the right. (Haematoxylin and eosin stain $\times 900$.)

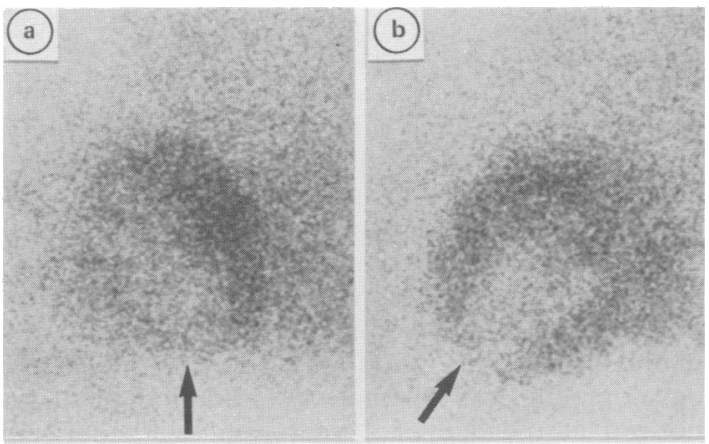

Fig 4 Thallium imaging at 26 years 6 months of age; $(a)=$ anterioposterior and $(b)=$ left anterior oblique $45^{\circ}$. Perfusion defect was observed from apex to posteroinferior portion (arrows). 
readmitted at the age of 25 years 11 months because of an appreciable progression of heart failure and pneumonia. His left ventricular ejection fraction decreased to $17 \%$ on echocardiography by the method of Pombo et al at age 26, and pronounced tricuspid and mild mitral valve regurgitation were recognised by colour flow mapping. He died at the age of 26 years and 9 months.

\section{Discussion}

The clinical signs observed in our patient meet the criteria of Emery-Dreifuss muscular dystrophy. ${ }^{5} \mathrm{We}$ observed his progressive deterioration by electrocardiography from an early cardiac conduction abnormality, manifest as first degree atrioventricular block, to sick sinus syndrome. In addition, development of cardiomyopathy was clearly disclosed by electrocardiography. Cardiac catheterisation and an echocardiogram, on the other hand, showed normal cardiac performance at age 17 when a permanent pacemaker was implanted. A specimen of the myocardium obtained at that time disclosed focal fibrosis, suggesting the presence of cardiomyopathy. Cardiomyopathy was also found by serial thallium imaging. Serial echocardiograms and gated blood pool scanning showed a rapid decrease of his left ventricular ejection fraction from the age 25 to 26 years and left ventricular hypokinesis. Although pronounced myocardial fibrosis has been described at necropsy ${ }^{6} 7$ or on myocardial biopsy, ${ }^{8}$ our patient clearly showed progressive cardiomyopathy in addition to defects in rhythm and conduction. A permanent cardiac pacemaker is effective in preventing sudden death due to profound bradycardia and syncope, but no effective treatment has been found at present for progressive cardiomyopathy. It is the cardiac involvement, not the systemic myopathy, that places the patient at risk. In future heart transplantation might be considered for the treatment of cardiomyopathy found in this syndrome if disorders of voluntary muscle were not considered to be an important irreversible dysfunction of major organs. ${ }^{9}$ Donor selection, however, might be a problem.

This study was supported by grant no $87 \mathrm{~A}-03$ from the National Center of Neurology and Psychiatry of the Ministry of Health and Welfare, Japan.

\section{References}

1 Emery AEH, Dreifuss FE. Unusual type of benign X-linked muscular dystrophy. J Neurol Neurosurg Psychiatry 1966;29: 338-42.

2 Waters DD, Nutter DO, Hopkins LC, Dorney ER. Cardiac features of an unusual $X$-linked humeroperoneal neuro-muscular disease. $N$ Engl J Med 1975;293:1017-22.

${ }^{3}$ Mandel W, Hayakawa H, Danzig R, Marcus HS. Evaluation of sino-atrial node function in man by overdrive suppression. Circulation 1971;44:59-66.

${ }^{4}$ Pombo JF, Troy BL, Russell RO Jr. Left ventricular volumes and ejection fraction by echocardiography. Circulation 1971;43: 480-90.

5 Rowland LP, Fetell M, Olarte M, Hays A, Singh N, Wanat FE. Emery-Dreifuss muscular dystrophy. Ann Neurol 1979;5:111-7.

${ }^{6}$ Thomas PK, Calne DB, Elliott CF. X-linked scapuloperoneal syndrome. J Neurol Neurosurg Psychiatry 1972;35:208-15.

${ }^{7}$ Iwashita H, Hara H, Mawatari S, Koga M, Fukuda K. Histological studies on the cardiac conducting system in patients with progressive muscular dystrophy (in Japanese). In: Nishitani $\mathrm{H}$, ed. 1985 annual research report on epidemiologic, clinical and therapeutic study of progressive muscular dystrophy. Tokyo, Japan: Ministry of Health and Welfare, 1985:169-73.

${ }^{8}$ Takamoto $\mathrm{K}$, Hirose $\mathrm{K}$, Uono M, Nonaka I. A genetic variant of Emery-Dreifuss disease. Arch Neurol 1984;41:1292-3.

9 Commerford PJ. Selection and management of the recipient. In: Cooper DKC, Lanza RP, eds. Heart transplantation. Lancaster: MTP Press, 1984:15-22.

Correspondence and requests for reprints to $\mathrm{Dr} M$ Yoshioka, Department of Pediatrics, Kobe General Hospital, 4-6 MinatojimaNakamachi, Chuo-ku, Kobe 650, Japan.

Accepted 26 October 1988 